\title{
Fluorescence laparoscopy imaging of pancreatic tumor progression in an orthotopic mouse model
}

\author{
Hop S. Tran Cao • Sharmeela Kaushal · Claudia Lee • \\ Cynthia S. Snyder · Kari J. Thompson · Santiago Horgan • \\ Mark A. Talamini $\cdot$ Robert M. Hoffman • Michael Bouvet
}

Received: 10 January 2010/ Accepted: 4 May 2010/Published online: 9 June 2010

(c) The Author(s) 2010. This article is published with open access at Springerlink.com

\begin{abstract}
Background The use of fluorescent proteins to label tumors is revolutionizing cancer research, enabling imaging of both primary and metastatic lesions, which is important for diagnosis, staging, and therapy. This report describes the use of fluorescence laparoscopy to image green fluorescent protein (GFP)-expressing tumors in an orthotopic mouse model of human pancreatic cancer.

Methods The orthotopic mouse model of human pancreatic cancer was established by injecting GFP-expressing MiaPaCa-2 human pancreatic cancer cells into the pancreas of 6-week-old female athymic mice. On postoperative day 14, diagnostic laparoscopy using both white and fluorescent light was performed. A standard laparoscopic system was modified by placing a 480-nm short-pass excitation filter between the light cable and the laparoscope in addition to using a 2-mm-thick emission filter. A camera was used that allowed variable exposure time and gain setting. For mouse laparoscopy, a 3-mm $0^{\circ}$ laparoscope was used. The mouse's abdomen was gently insufflated to $2 \mathrm{~mm} \mathrm{Hg}$ via a 22-gauge angiocatheter. After laparoscopy, the
\end{abstract}

H. S. Tran Cao · K. J. Thompson · S. Horgan ·

M. A. Talamini - R. M. Hoffman - M. Bouvet

Department of Surgery, University of California-San Diego,

San Diego, CA, USA

S. Kaushal - C. S. Snyder · R. M. Hoffman - M. Bouvet ( $\square)$ Moores UCSD Cancer Center, 3855 Health Sciences Drive

\#0987, La Jolla, CA 92093-0987, USA

e-mail: mbouvet@ucsd.edu

C. Lee

UVP LLC, Upland, CA, USA

R. M. Hoffman

AntiCancer, Inc., San Diego, CA, USA animals were sacrificed, and the tumors were collected and processed for histologic review. The experiments were performed in triplicate.

Results Fluorescence laparoscopy enabled rapid imaging of the brightly fluorescent tumor in the pancreatic body. Use of the proper filters enabled simultaneous visualization of the tumor and the surrounding structures with minimal autofluorescence. Fluorescence laparoscopy thus allowed exact localization of the tumor, eliminating the need to switch back and forth between white and fluorescence lighting, under which the background usually is so darkened that it is difficult to maintain spatial orientation.

Conclusion The use of fluorescence laparoscopy permits the facile, real-time imaging and localization of tumors labeled with fluorescent proteins. The results described in this report should have important clinical potential.

Keywords Green fluorescent protein - Laparoscopy · Orthotopic model $\cdot$ Nude mice $\cdot$ Pancreatic cancer

Accurate staging is a crucial step in establishing the appropriate therapeutic approach for patients with pancreatic cancer. Curative resection can be achieved in only 10 $20 \%$ of newly diagnosed cases [1]. Despite improvements in computed tomography (CT), magnetic resonance imaging (MRI), and endoscopic ultrasound (EUS), up to $36 \%$ of patients deemed to have resectable disease based on preoperative imaging with these techniques are found at staging laparoscopy to have unresectable disease $[2,3]$.

Although staging laparoscopy has been demonstrated to improve the diagnostic yield of peritoneal and liver metastases otherwise undetectable by preoperative imaging, it is not sufficiently accurate [2-4]. Indeed, studies examining the accuracy of staging laparoscopy in 
predicting the resectability of peripancreatic tumors showed false-negative rates of $18-26 \%$ [5, 6]. Whereas some authors report improved accuracy with the addition of laparoscopic ultrasound [7], others show that falsenegative rates can remain as high as $20 \%$ [8]. Given these problems, efforts to improve the accuracy of staging laparoscopy further are necessary.

Because regular white light can be strongly absorbed and scattered by normal surrounding tissue, metastatic foci in the peritoneum and on the liver, which often are small and flat, can go undetected under standard examination. The photosensitizer 5-aminolevulinic acid (ALA) and its metabolite protoporphyrin IX (PPIX) have been used to fluorescently label a wide range of tumors including ovarian, renal, and various gastrointestinal malignancies for fluorescence laparoscopy [9-12]. However, the resolution achieved with this method needs improvement.

Our laboratory pioneered the use of fluorescent proteins for imaging tumors in mouse models [13] and has developed this technology for fluorescence-guided surgery [14]. In this study, we applied fluorescent protein-based imaging to laparoscopy of human pancreatic cancer in an orthotopic mouse model.

Our study aimed to develop fluorescence laparoscopy that strongly detects the bright signal from a fluorescently labeled tumor while still allowing adequate visualization of the background. Such technology would be instrumental in future clinical applications of fluorescence laparoscopy, especially for surgical navigation.

\section{Materials and methods}

\section{Cell culture}

Human MiaPaCa-2 cells were stably transduced to express the green fluorescent protein (GFP) as previously described $[15,16]$. These cells were maintained in Dulbecco's modified Eagle medium (DMEM) (Gibco-BRL, Grand Island, NY, USA) supplemented with $10 \%$ fetal bovine serum (Hyclone, Logan, UT, USA), penicillin/streptomycin (Gibco-BRL), sodium pyruvate (Gibco-BRL), sodium bicarbonate (Cellgro, Manassas, VA, USA), L-glutamine (Gibco-BRL), and minimal essential medium (MEM) nonessential amino acids (Gibco-BRL). Cells were incubated at $37^{\circ} \mathrm{C}$ with $5 \%$ carbon dioxide $\left(\mathrm{CO}_{2}\right)$.

Animal care

Female athymic $n u / n u$ nude mice were maintained in a barrier facility on high-efficiency particulate air (HEPA)filtered racks. The animals were fed with autoclaved laboratory rodent diet (Teckland LM-485; Western Research
Products, Orange, CA, USA). All surgical procedures were performed with the animals under anesthesia using an intramuscular injection of $0.02 \mathrm{ml}$ of a $50 \%$ ketamine, $38 \%$ xylazine, and $12 \%$ acepromazine maleate solution. The animals were sacrificed by injecting $0.05 \mathrm{ml}$ of the same solution, followed by cervical dislocation. All animal studies were approved by the UCSD Institutional Animal Care and Use Committee (IACUC) and conducted in accordance with the principles and procedures outlined in the National Institutes of Health (NIH) Guide for the Care and Use of Animals.

Orthotopic pancreatic cancer model

MiaPaCa-2-GFP cells were harvested by trypsinization and washed three times with serum-free medium. Viability was verified to be greater than $95 \%$ using the Vi-Cell XR automated cell viability analyzer (Beckman Coulter, Brea, CA, USA). The cells were resuspended at concentrations of $1 \times 10^{6}$ per $20 \mu \mathrm{l}$ of serum-free medium and placed on ice before surgery.

Orthotopic implantation was performed in 6-week-old female nude mice by first making a 6- to 10 -mm transverse incision on the left flank of the mouse through the skin and peritoneum. The tail of the pancreas then was exposed through this incision. Pancreatic cancer cells $\left(1 \times 10^{6}\right)$ were injected into the pancreatic tail, which was subsequently returned into the abdomen. The incision was closed in two layers using 6.0 Ethibond nonabsorbable suture (Ethicon Inc., Somerville, NJ, USA).

Fluorescence laparoscopy

An ideal fluorescence laparoscope should possess the following properties: first, it should maximize the fluorescence signal of the tumor to facilitate its easy and rapid imaging. Second, it should provide a clear view of the background and surrounding tissues to allow maintenance of spatial orientation. This second aspect has special importance when the scope is used in a therapeutic rather than a strictly diagnostic capacity. To achieve these criteria, we modified a standard laparoscopic system in the following manner (Fig. 1): the excitation light source, a 300-W Xenon lamp (Stryker, Kalamazoo, MI, USA), was filtered by a 480-nm interference short-pass filter. To avoid heat damage to the filter, this excitation filter was placed at the end of the optical fiber, which delivers the light to the laparoscope. An emission filter, whose selection is described below, was also placed between the laparoscope and the camera. A MultiCam 310C camera (UVP, Upland, CA, USA), which allows variable exposure time and gain setting in the controlling software (VisionWorks LS, UVP), was used. To meet our objective of dual functionality 
Fig. 1 Fluorescence laparoscope for visualization and localization of green fluorescent protein (GFP)labeled tumors in mice. A laparoscopic tower was modified to achieve a fluorescence light mode that would permit detection of fluorescence signals while still allowing visualization of the background tissue. A 480-nm short-pass filter was placed between the light cable and the laparoscope. A GG495 glass filter was placed between the laparoscope and a camera that controls exposure time and gain. These parameters were set to $110 \mathrm{~ms}$ and 97 , respectively, for the purpose of our experiment

through live video, the exposure time was set at $110 \mathrm{~ms}$, and the gain was set to 97 .

The emission filter was chosen from three possible options that match in the emission band of GFP at $520 \mathrm{~nm}$ (a 515-nm long-pass interference filter, a Schott OG515 glass filter, or a Schott GG495 glass filter) after the absorption spectra of their respective combinations with the 480-nm excitation filter had been obtained. The background transmission ratio was calculated from summing the absorption spectra of the filter combinations and translating the combined absorbance $(\mathrm{A})$ to transmission $\left(10^{-\mathrm{A}}\right)$. The results indicated that the 2-mm-thick Schott GG495 glass filter was the preferred emission filter (see Results section). This particular filter was preferable because, unlike interference filters that typically have optical density (OD) 4 (i.e. leaking only $10^{-4}$ of light outside the allowed spectrum) or higher capability in blocking the background, it allows $1 \%$ of the background to leak through. This design affords visualization of the nonfluorescent tissue for navigation while enhancing the fluorescence signal of the tumor.

\section{Mouse laparoscopy}

Two weeks after orthotopic implantation of the MiaPaCa2-GFP cells, the mice were anesthetized before laparoscopy. Under sterile conditions, a 22-gauge angiocatheter was gently introduced into the abdominal cavity at an angle to avoid injury to the underlying bowel. The catheter was secured to the abdominal wall with a suture and connected to the insufflation tubing of the laparoscopic tower. Insufflation was first initiated at $1 \mathrm{~mm} \mathrm{Hg}$, then augmented to a final pressure of $2 \mathrm{~mm} \mathrm{Hg}$.

Next, a small incision was made higher up in the abdomen, through which a 3-mm $0^{\circ}$ Karl-Storz laparoscope (Karl-Storz GmbH \& Co., Tuttlingen, Germany) was introduced. A purse-string suture was placed around this incision to prevent its widening and to maintain proper insufflation (Fig. 2). Due to the limitations presented by the small size of the mice, no other ports were placed. At termination of laparoscopy, the mice were sacrificed for tumor collection.

Tissue histology

At necropsy, the fluorescent tumor was collected, placed in Bouin's solution, sectioned, and subsequently placed on slides for histology with hematoxylin and eosin (H\&E) staining. The slides were reviewed by a pathologist to confirm the identity of the fluorescent lesion.

\section{Data processing}

Images obtained during laparoscopy were not processed in any way. Representative frames are presented. Histology images were processed for brightness and contrast using Photoshop Element 4 (Adobe Systems Inc., San Jose, CA, USA).

\section{Results}

Two weeks after orthotopic implantation of MiaPaCa-2GFP human pancreatic cancer cells, female athymic mice were anesthetized before undergoing a diagnostic laparoscopy under both white and fluorescence modes. By this time, all the animals had tumors that measured approximately $1 \mathrm{~cm}$ in diameter. For fluorescence laparoscopy, insufflation to $2 \mathrm{~mm} \mathrm{Hg}$ allowed ample distention of the abdominal wall to enable adequate visualization and navigation within the abdomen of the mice. 


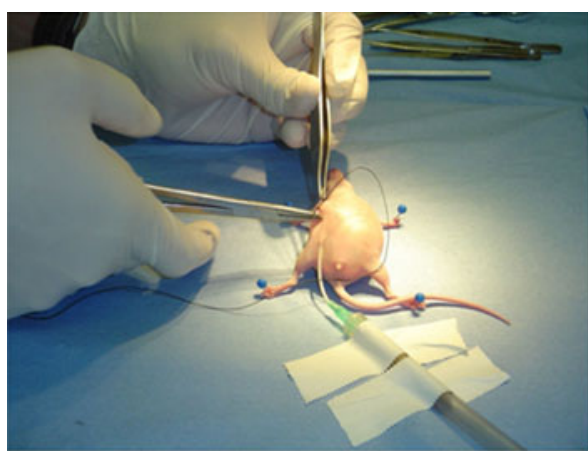

TROCHAR PLACEMENT

Fig. 2 Mouse laparoscopy. To achieve laparoscopy in the mouse, a 22 -gauge angiocatheter was inserted into the abdominal cavity and attached to the insufflation tubing of the laparoscopic tower. The

Because peak excitation for the GFP occurs at $488 \mathrm{~nm}$, a 480-nm short-pass excitation filter was used. Three different emission filters were tested that all achieved excellent and equivalent background suppression in the emission band of GFP at $520 \mathrm{~nm}$, which serves to create the best contrast between fluorescence and background. Additionally, the distinguishing advantage of an ideal emission filter would be preservation of sufficient contrast while enough of the background is allowed to leak through to maintain spatial orientation.

Spectral analysis of combinations of the 480-nm excitation filter with each of the three emission filters tested demonstrated that at wavelengths shorter than $520 \mathrm{~nm}$, the GG495 filter allowed the best transmission of background light while achieving the same degree of contrast between fluorescence and background (Fig. 3).

When either the OG515 or 515LP emission filters were used, the fluorescent tumor became visible. However, the background became so darkened that it was impossible to pinpoint the location of the tumor (Fig. 4a, b). To visualize the surrounding tissue and locate the tumor, the laparoscope would have to be switched back and forth between white and fluorescence modes. The GG495 filter overcame this problem (Fig. 4c). The combination of the Xenon lamp, the 480-nm short-pass excitation filter, and the GG495 long-pass emission filter produced a spectrum that not only provided a sufficient background but also resulted in proper color balance. By using a camera with adjustable exposure time and gain, these two parameters can be optimized to compensate for the lack of intensity from the Xenon lamp.

For the purpose of our experiment, an exposure time of $110 \mathrm{~ms}$ and a gain of 97 afforded clear visualization of both the fluorescent tumor and the surrounding structures. The increasing noise due to increased gain was dynamic and did not interfere with the diagnostic purpose. The

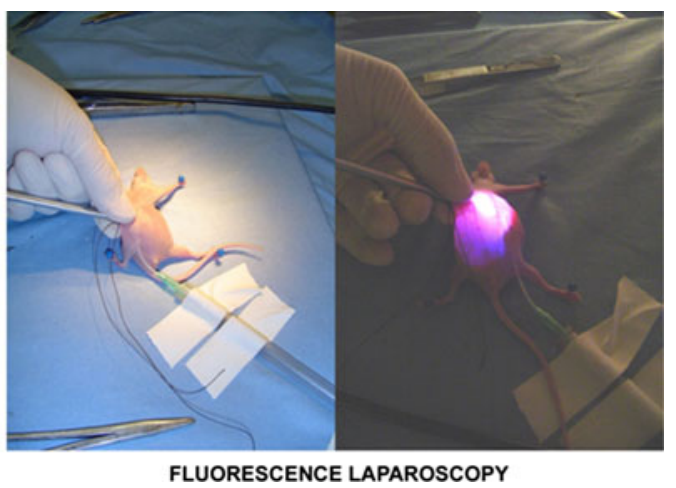

abdomen was insufflated to $2 \mathrm{~mm} \mathrm{Hg}$, and a 3-mm $0^{\circ}$ laparoscope was introduced into the abdomen for diagnostic purposes. The laparoscope was secured with a purse-string suture

exposure time, however, had to be kept shorter than the time frame of typical biologic reactions, such as the interval of heart beats, to remain useful for navigation.

With this proper combination of instruments and light parameters, fluorescence laparoscopy permitted rapid and easy identification of the fluorescent tumor while still permitting visualization of the surrounding tissue. In fact, the GFP-expressing tumor was sufficiently bright to enable its instant localization (Fig. 5). Navigation through the abdominal cavity under this fluorescent mode was straightforward because we were able to maintain an adequate view of the surrounding structures.

At termination of laparoscopy, the mice were sacrificed, and the identified lesions were collected for histology. The use of H\&E stain confirmed that these fluorescent lesions were indeed pancreatic adenocarcinoma tumors (Fig. 6).

\section{Discussion}

Staging laparoscopy has a demonstrated role in the evaluation of certain gastrointestinal malignancies, including gastric cancer [17] and pancreatic cancer [4]. The detection of a cancerous lesion on the surface of the peritoneum or the liver in a minimally invasive way can spare the patient with metastatic disease from an unnecessary and costly celiotomy, fraught with potential complications. Unfortunately, standard laparoscopy can bear false-negative rates as high as $26 \%$, underscoring the need to improve its diagnostic yield [6].

Several authors have previously described the application of fluorescence technology to this endeavor, relying on ALA-induced PPIX to highlight potentially malignant lesions [9-12]. Although the results have been impressive, ALA is not cancer-specific and can at times fluorescently label benign lesions $[10,11]$. Labeling that is cancer- 


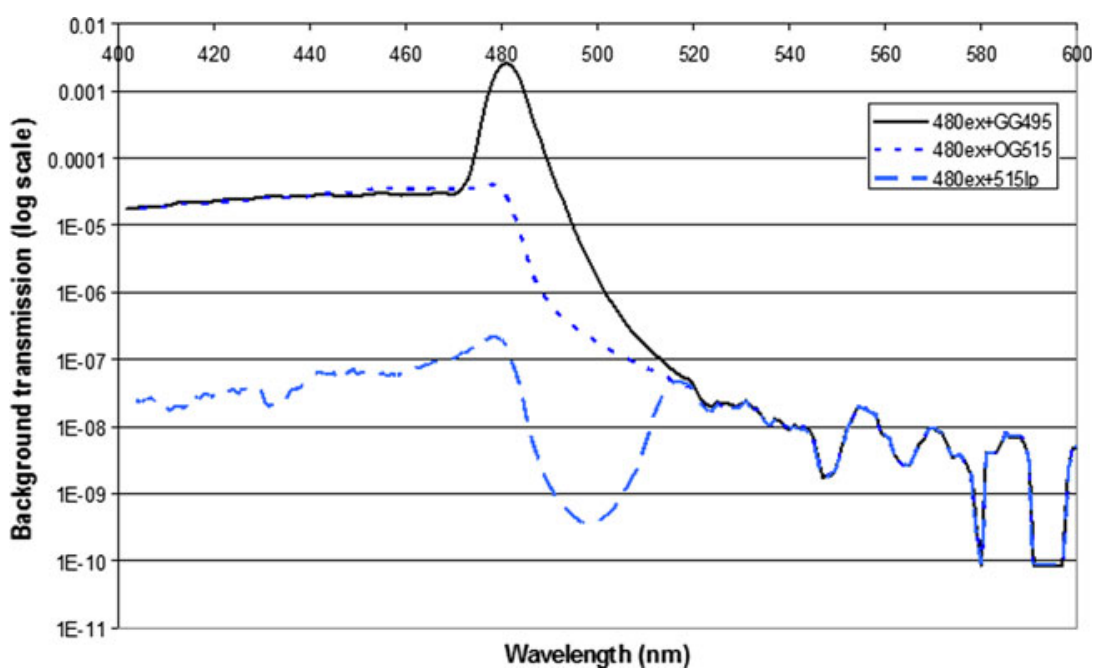

Fig. 3 Spectral analysis of filter combinations of the 480-nm excitation filter and three potential emission filters. At $520 \mathrm{~nm}$ within the emission band of green fluorophores (green fluorescent protein [GFP], Alexa-488, fluorescein), all three combinations performed equally well. However, at shorter wavelengths, they differed significantly, resulting in different background transmission capabilities.
The 515-nm long-pass interference filter almost completely blocked background transmission, whereas the two glass filters (GG495 and OG515) achieved a more desirable signal-to-background ratio. The background ratio of GG495 was appropriate for generating a non zero background for navigation and signal detection without fully saturating the charge-coupled device (CCD) which can cause loss of detail
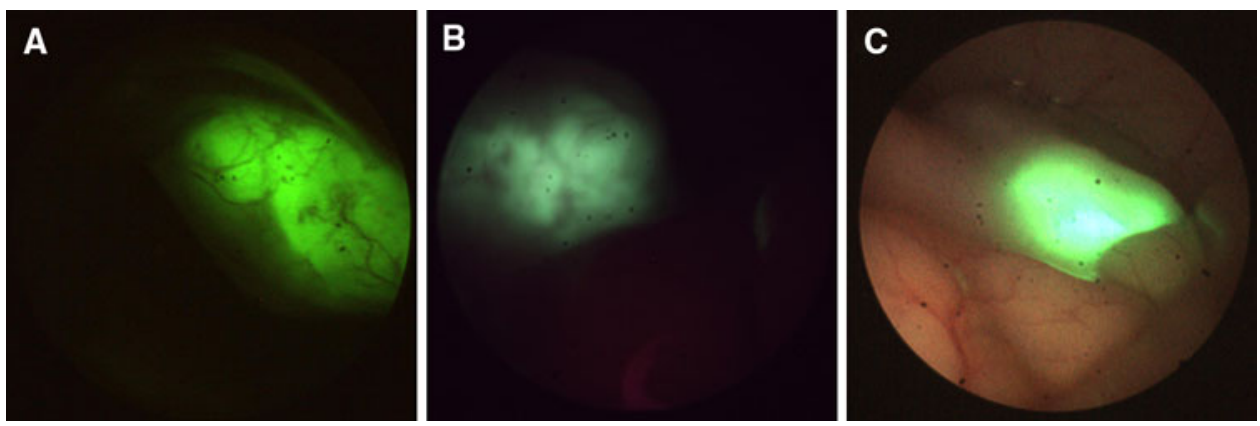

Fig. 4 Fluorescence signal from a green fluorescent protein (GFP)expressing pancreatic tumor as seen with three different emission filters. A When an interference filter was used, the fluorescence signal was strong, but the background was almost completely blocked out and appeared nearly uniformly black. B Using the OG515 glass filter instead, slightly more background came through, although it was still insufficient for navigation. Also, the color balance with this filter was not optimal. C The use of the GG495 glass filter achieved a good color balance and adequate visualization of the background while maintaining good signal-background contrast
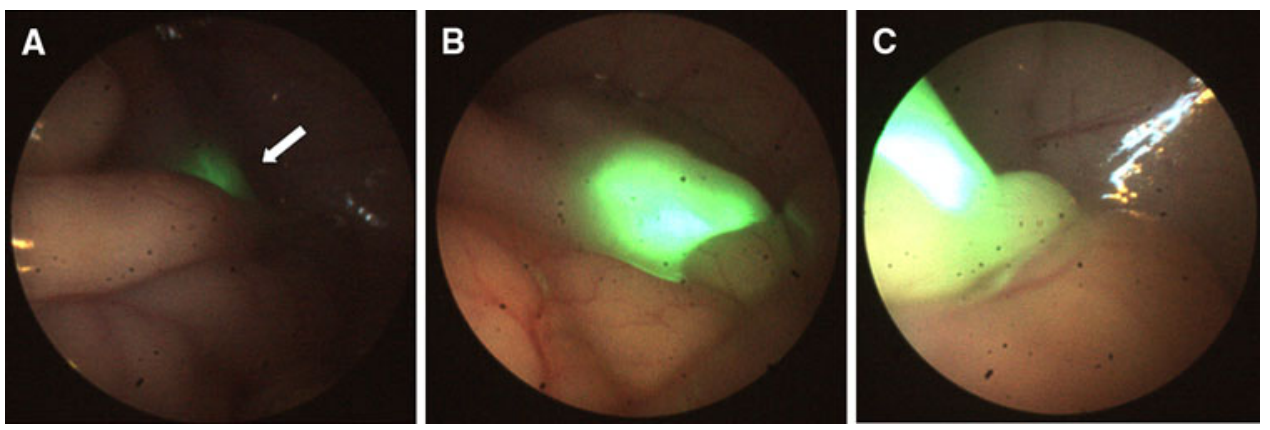

Fig. 5 Fluorescence laparoscopy at different angles and distances in a mouse with orthotopically implanted green fluorescent protein (GFP)-expressing pancreatic cancer. Due to the fluorescence of the tumor, a green glow (arrow) was quickly detected at entry into the abdominal cavity (A) which easily led to the exact localization of the tumor within the body of the pancreas $(\mathbf{B}, \mathbf{C})$. The fluorescence signal of the tumor was easily seen. The surrounding structures, including the bowel and its vessels remained clearly visible as well permitting facile navigation within the abdominal cavity without the need to flip between a fluorescence mode and a white light mode 


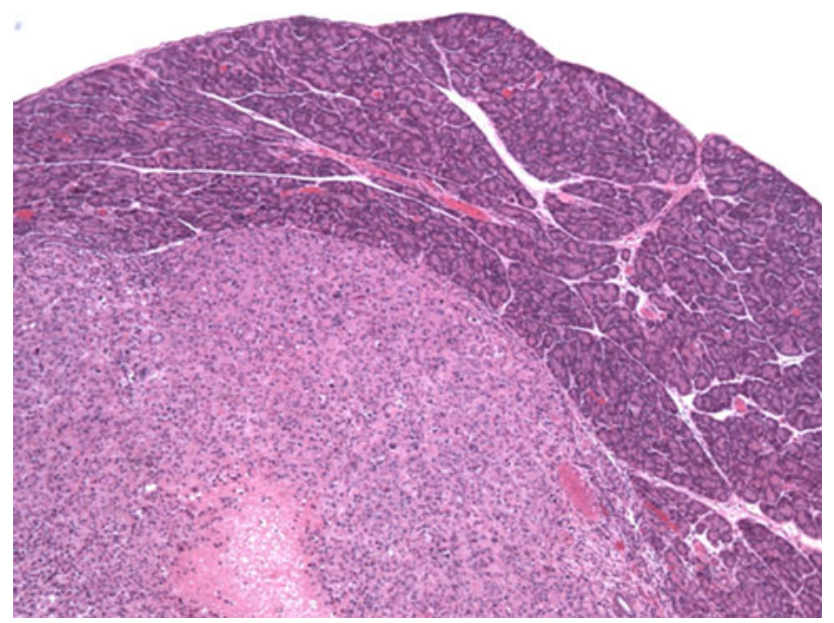

Fig. 6 Histologic image of a resected green fluorescent protein (GFP)-expressing, orthotopic tumor implant. Hematoxylin and eosin (H\&E)-stained sections demonstrated the expansile tumor formed by orthotopically implanted GFP-expressing human pancreatic carcinoma cells (MiaPaCa-2) within the body of the mouse pancreas. The poorly differentiated tumor, surrounded by a variable thickness of normal pancreatic parenchyma, showed scattered foci of zonal necrosis, as seen in the lower edge of the image

specific and selective could be of great value. Furthermore, the fluorescence signal elicited from PPIX can be intrinsically weak. In one study using ALA in neurosurgery, fluorescence of the glioma evaluated was only $25 \%$ greater than that of the normal brain tissue and was surpassed by that of the periosteum and skin [18]. This weakness of the fluorescence signal, when translated to the laparoscopic setting, would require a fluorescence mode that inevitably darkens the background significantly.

Likewise, Adusumilli et al. [19] have previously described and used GFP-guided fluorescence laparoscopy for tumor detection using a replication-competent herpes simplex virus to label the tumors with GFP. In that study, the investigators alternated between the use of white light and fluorescence light to spatially localize the tumor. This could pose potential disadvantages, especially if careful surgical navigation is required around critical structures. As such, the value of fluorescence laparoscopy is diminished when the strength of the fluorescent signal is enhanced at the expense of the visibility of the background.

With this in mind, we developed a laparoscopic fluorescence mode that allows facile and rapid identification and localization of a fluorescently labeled tumor while still maintaining adequate visualization of the surrounding tissue. The clear advantage that it offers is the ability to navigate within the abdominal cavity without losing spatial orientation, something that the standard ultraviolet light described in previous fluorescence laparoscopy reports cannot do.

Two main issues govern the efficacy of fluorescence laparoscopy. First, the right filter sets must be used to permit proper excitation of the fluorescent tumor and detection of its signal while still allowing visualization of the surrounding tissue for navigation. Second, even with the proper filter set in place, the exposure time of the fluorophore to the exciting light must be lengthened to enhance the brightness of the fluorescence signal, which would otherwise be rather weak. In addition, the gain must be adjusted to optimize visualization of both the tumor and surrounding tissues. By modifying a standard laparoscope with a set of excitation and emission filters that allow some background leakage of bright light without compromising signal-background contrast and by adjusting camera-controlled parameters such as exposure time and gain, we were able to quickly and easily detect a GFP-expressing tumor in the body of the mouse's pancreas.

Although we have chosen to use pancreatic cancer cells previously engineered to highly express GFP in vivo for the purpose of this study, it must be pointed out that a number of methods have been used to fluorescently label intrinsic, existing tumors. Our laboratory has pioneered several of these techniques, including the use of antibodies against tumor antigens such as carcinoembryonic antigen (CEA) or CA19-9 to deliver fluorophores to pancreatic cancer lesions [20, 21]. Because CEA and CA19-9 are expressed in up to $98 \%$ and $91 \%$ of pancreatic ductal adenocarcinomas, respectively [22], the clinical application of fluorophoreconjugated antibodies directed against them should allow identification of almost all pancreatic adenocarcinoma lesions. Fluorescence laparoscopy of tumors labeled with GFP or fluorescent antibodies should be a powerful tool in the diagnosis and staging of pancreatic cancer.

Acknowledgments This work was supported in part by grants CA109949 and CA132971 from the National Cancer Institute and American Cancer Society RSG-05-037- 01-CCE (to M.B.), T32 training grant CA121938 (to H.S.T.C.), and National Cancer Institute Grant CA103563 (to AntiCancer, Inc.).

Disclosures Hop S. Tran Cao, Sharmeela Kaushal, Claudia Lee, Cynthia S. Snyder, Kari J. Thompson, Santiago Horgan, Mark A. Talamini, Robert M. Hoffman, Michael Bouvet have no financial interests or ties to disclose.

Open Access This article is distributed under the terms of the Creative Commons Attribution Noncommercial License which permits any noncommercial use, distribution, and reproduction in any medium, provided the original author(s) and source are credited.

\section{References}

1. Sener SF, Fremgen A, Menck HR, Winchester DP (1999) Pancreatic cancer: a report of treatment and survival trends for 100 , 313 patients diagnosed from 1985-1995, using the National Cancer Database. J Am Coll Surg 189:1-7

2. Conlon KC, Dougherty E, Klimstra DS, Coit DG, Turnbull AD, Brennan MF (1996) The value of minimal access surgery in the 
staging of patients with potentially resectable peripancreatic malignancy. Ann Surg 223:134-140

3. Jimenez RE, Warshaw AL, Rattner DW, Willett CG, McGrath D, Fernandez-del Castillo C (2000) Impact of laparoscopic staging in the treatment of pancreatic cancer. Arch Surg 135:409-414 (discussion 414-405)

4. Stefanidis D, Grove KD, Schwesinger WH, Thomas CR Jr (2006) The current role of staging laparoscopy for adenocarcinoma of the pancreas: a review. Ann Oncol 17:189-199

5. Arnold JC, Neubauer HJ, Zopf T, Schneider A, Benz C, Adamek HE, Riemann JF (1999) Improved tumor staging by diagnostic laparoscopy. Z Gastroenterol 37:483-488

6. Nieveen van Dijkum EJ, Romijn MG, Terwee CB, de Wit LT, van der Meulen JH, Lameris HS, Rauws EA, Obertop H, van Eyck CH, Bossuyt PM, Gouma DJ (2003) Laparoscopic staging and subsequent palliation in patients with peripancreatic carcinoma. Ann Surg 237:66-73

7. Minnard EA, Conlon KC, Hoos A, Dougherty EC, Hann LE, Brennan MF (1998) Laparoscopic ultrasound enhances standard laparoscopy in the staging of pancreatic cancer. Ann Surg 228:182-187

8. Doran HE, Bosonnet L, Connor S, Jones L, Garvey C, Hughes M, Campbell F, Hartley M, Ghaneh P, Neoptolemos JP, Sutton R (2004) Laparoscopy and laparoscopic ultrasound in the evaluation of pancreatic and periampullary tumours. Dig Surg 21:305-313

9. Gahlen J, Prosst RL, Pietschmann M, Haase T, Rheinwald M, Skopp G, Stern J, Herfarth C (2002) Laparoscopic fluorescence diagnosis for intraabdominal fluorescence targeting of peritoneal carcinosis experimental studies. Ann Surg 235:252-260

10. Hoda MR, Popken G (2009) Surgical outcomes of fluorescenceguided laparoscopic partial nephrectomy using 5-aminolevulinic acid-induced protoporphyrin IX. J Surg Res 154:220-225

11. Loning M, Diddens H, Kupker W, Diedrich K, Huttmann G (2004) Laparoscopic fluorescence detection of ovarian carcinoma metastases using 5-aminolevulinic acid-induced protoporphyrin IX. Cancer 100:1650-1656

12. Zopf T, Schneider AR, Weickert U, Riemann JF, Arnold JC (2005) Improved preoperative tumor staging by 5 -aminolevulinic acid-induced fluorescence laparoscopy. Gastrointest Endosc 62:763-767
13. Hoffman RM (2005) The multiple uses of fluorescent proteins to visualize cancer in vivo. Nat Rev Cancer 5:796-806

14. Kishimoto H, Zhao M, Hayashi K, Urata Y, Tanaka N, Fujiwara T, Penman S, Hoffman RM (2009) In vivo internal tumor illumination by telomerase-dependent adenoviral GFP for precise surgical navigation. Proc Natl Acad Sci USA 106:14514-14517

15. Bouvet M, Wang J, Nardin SR, Nassirpour R, Yang M, Baranov E, Jiang P, Moossa AR, Hoffman RM (2002) Real-time optical imaging of primary tumor growth and multiple metastatic events in a pancreatic cancer orthotopic model. Cancer Res 62:15341540

16. Bouvet $M$, Yang $M$, Nardin S, Wang $X$, Jiang $P$, Baranov E, Moossa AR, Hoffman RM (2000) Chronologically specific metastatic targeting of human pancreatic tumors in orthotopic models. Clin Exp Metastasis 18:213-218

17. Lowy AM, Mansfield PF, Leach SD, Ajani J (1996) Laparoscopic staging for gastric cancer. Surgery 119:611-614

18. Ciburis A, Gadonas D, Gadonas R, Didziapetriene J, Gudinaviciene I, Grazeliene G, Kaskelyte D, Piskarskas A, Ksauminas K, Smilgevicius V, Sukackaite A (2003) 5-Aminolevulinic acid induced protoporphyrin IX fluorescence for detection of brain tumor cells in vivo. Exp Oncol 25:51-54

19. Adusumilli PS, Eisenberg DP, Stiles BM, Chung S, Chan MK, Rusch VW, Fong Y (2006) Intraoperative localization of lymph node metastases with a replication-competent herpes simplex virus. J Thorac Cardiovasc Surg 132:1179-1188

20. Kaushal S, McElroy MK, Luiken GA, Talamini MA, Moossa AR, Hoffman RM, Bouvet M (2008) Fluorophore-conjugated antiCEA antibody for the intraoperative imaging of pancreatic and colorectal cancer. J Gastrointest Surg 12:1938-1950

21. McElroy M, Kaushal S, Luiken GA, Talamini MA, Moossa AR, Hoffman RM, Bouvet M (2008) Imaging of primary and metastatic pancreatic cancer using a fluorophore-conjugated antiCA19-9 antibody for surgical navigation. World J Surg 32:10571066

22. Yamaguchi K, Enjoji M, Tsuneyoshi M (1991) Pancreatoduodenal carcinoma: a clinicopathologic study of 304 patients and immunohistochemical observation for CEA and CA19-9. J Surg Oncol 47:148-154 